\title{
Intrathecal triamcinolone acetonide exerts anti-inflammatory effects on Lewis rat experimental autoimmune neuritis and direct anti-oxidative effects on Schwann cells
}

Kalliopi Pitarokoili*, Melissa Sgodzai, Thomas Grüter, Hussein Bachir, Jeremias Motte, Björn Ambrosius, Xiomara Pedreiturria, Min-Suk Yoon and Ralf Gold

\begin{abstract}
Background: Corticosteroids dominate in the treatment of chronic autoimmune neuropathies although long-term use is characterized by devastating side effects.

Methods: We introduce the intrathecal application of the synthetic steroid triamcinolone (TRIAM) as a novel therapeutic option in experimental autoimmune neuritis in Lewis rats

Results: After immunization with neuritogenic P2 peptide, we show a dose-dependent therapeutic effect of one intrathecal injection of 0.3 or $0.6 \mathrm{mg} / \mathrm{kg}$ TRIAM on clinical and electrophysiological parameters of neuritis with a lower degree of inflammatory infiltrates (T cells and macrophages) and demyelination in the sciatic nerve. In vitro studies in Schwann cell cultures showed an increased expression of IL-1 receptor antagonist and reduced expression of Toll-like receptor 4 after incubation with TRIAM as well as a protective effect of TRIAM against oxidative stress after $\mathrm{H}_{2} \mathrm{O}_{2}$ exposure.

Conclusion: Intrathecal TRIAM application could be a novel immunomodulatory and potentially neuroprotective option for autoimmune neuropathies with a direct effect on Schwann cells.
\end{abstract}

Keywords: Autoimmune neuropathies, Triamcinolone, Experimental autoimmune neuritis, Autoimmunity, Inflammation, Immunotherapy

\section{Introduction}

Triamcinolone acetonide (TRIAM) is an intermediate -acting synthetic glucocorticoid, which as a sustained-release crystal suspension is suitable for sub-lesional injections for isolated psoriasis or discoid lupus erythematosus, intra-articular injections for the treatment of chronic inflammatory joint diseases, and intravitreal applications as it reduces macular edema and angiogenesis in eyes with artificial lenses $[1,2]$.

Regarding autoimmune diseases of the central nervous system (CNS), repeated intrathecal application of the

* Correspondence: Kalliopi.Pitarokoili@ruhr-uni-bochum.de Department of Neurology, St. Josef Hospital, Ruhr-University Bochum, Gudrunstr. 56, 44791 Bochum, Germany sustained release TRIAM (40-80 mg every other day for one to three injections) is performed since the 1970s in specialized centers in Germany for primary and secondary progressive multiple sclerosis patients and improves, according to observational trials in up to 161 patients, the maximum walking distance, spasticity, and occupational deficits of the upper extremities [3-9]. Three months after repeated application of TRIAM in the cerebrospinal fluid (CSF), an elevated steroid level could still be found. Although data from large-scale clinical trials are missing, intrathecal TRIAM application is still used from specialized centers as an off-label treatment [4].

(C) The Author(s). 2019 Open Access This article is distributed under the terms of the Creative Commons Attribution 4.0 International License (http://creativecommons.org/licenses/by/4.0/), which permits unrestricted use, distribution, and reproduction in any medium, provided you give appropriate credit to the original author(s) and the source, provide a link to the Creative Commons license, and indicate if changes were made. The Creative Commons Public Domain Dedication waiver (http://creativecommons.org/publicdomain/zero/1.0/) applies to the data made available in this article, unless otherwise stated. 
Our group has previously shown that intrathecal therapeutic application of human immunoglobulins in the context of experimental autoimmune neuritis (EAN), the animal model of dysimmune neuropathies such as Guillain-Barré syndrome (GBS), or the chronic inflammatory demyelinating polyradiculoneuropathy (CIDP) achieves a dose-dependent improvement of clinical signs, which correlate with a histological reduction of inflammatory infiltrates in the sciatic nerves and of complement activation in the sciatic nerve [10].

Corticosteroids are used as first-line therapy in the form of pulsed intravenous methylprednisolone or oral long-term prednisolone treatment for patients with CIDP and improve sensory symptoms and painful paresthesia. Unfortunately, their therapeutic benefits are limited by side effects such as osteoporosis, abdominal obesity, glaucoma, diabetes mellitus, and hypertension (Cushing's syndrome) [2].

Intrathecal application route has not yet been investigated for TRIAM in autoimmune diseases of the peripheral nervous system (PNS) although the first sites of inflammation and increase of blood-nerve barrier permeability are the proximal nerve roots, as indicated by the early intrathecal protein increase found in these patients $[11,12]$.

According to the published literature, TRIAM mediates a combination of anti-inflammatory, anti-oedematous, anti-proliferative, anti-angiogenetic, and neuroprotective effects, which could be very crucial for autoimmune diseases of the PNS. The anti-inflammatory effects of TRIAM have been widely investigated and are mediated after binding to the glucocorticoid (GR) nuclear receptors, which is widely expressed in neurons and Schwann cells. Upon binding TRIAM, the receptors undergo a conformational change and translocate in the nucleus to mediate gene transcription and induce an anti-inflammatory potential for example through inhibiting cytokine release (e.g., TNF $\alpha$ and IL-1 $\beta$ ). GR can also signal through nongenomic pathways which occur rapidly and do not require transcriptional changes $[13,14]$.

Intrathecal TRIAM was also suggested to have neuroprotective efficacy on infarction volume in acute focal cerebral ischemia in rats. Compared with controls (18.2\%), infarction volume was significantly reduced using TRIAM injection into the cisterna magna (13.4\%) [15].

In this study, we describe for the first time the immunomodulatory effects of triamcinolone in vivo applied intrathecally in the Lewis rat model of EAN. Moreover, this study describes indirect neuroprotective mechanisms in vitro in the context of Schwann cell culture. Therefore, we present the first report of an effective and dose-saving route of administration of TRIAM for dysimmune neuropathies.

\section{Materials and methods \\ Antigens}

The neuritogenic P2 peptide, corresponding to amino acids 53-78 of rat myelin P2 protein, was synthesized by Dr. Rudolf Volkmer from Charité Universitätsmedizin (Berlin, Germany).

\section{Triamcinolone}

Triamcinolone acetonide (Volon A, Bristol-Meyers Squibb, New York, NY, USA, active ingredient 16a.17-Dimethyl-

methylendioxy-9-fluor-11b.21-dihydroxy-1.4-pregnadien

- 3.20-dion) supplied in sodium chloride solution equivalent to $10 \mathrm{mg}$ TRIAM $/ \mathrm{ml}$ or $40 \mathrm{mg}$ TRIAM $/ \mathrm{ml}$ was used for all experiments.

\section{Study design-disease induction and clinical score assessment}

A total of 45 female Lewis rats were randomized for the therapeutic concept as described in the following section. The rats were 6-8 weeks old; they were purchased from Charles River Co. (Sulzfeld, Germany) and weighed 160$180 \mathrm{~g}$ when used for the following experiments. They were anesthetized by $1.5-2.0 \%$ halothane in oxygen. They were immunized by subcutaneous injection of $250 \mu \mathrm{g} \mathrm{P}_{53-78}$ peptide in phosphate-buffered saline (PBS) into the root of the tail, emulsified in an equal volume of complete Freund's adjuvant (CFA) containing Mycobacterium tuberculosis $(1 \mathrm{mg} / \mathrm{ml})$ H37RA (Difco, Detroit, MI, USA). Animals were weighted and scored for disease severity daily by two independent, blinded investigators. Disease severity was assessed clinically employing a scale ranging from 0 to 10 originally described by Enders et al. [16]: 0, normal; 1, less lively; 2 , impaired righting/limb tail; 3 , absent righting; 4, atactic gait, abnormal position; 5 , mild paraparesis; 6 , moderate paraparesis; 7 , severe paraplegia; 8 , tetraparesis; 9, moribund; 10, death. All experiments were reviewed and approved by the North-Rhine-Westphalia, Germany authorities for animal experimentation (TVA 84-02.04.2017-A023).

\section{Intrathecal treatment with triamcinolone and dosage rationale}

The animals were anesthetized by $1.5-2.0 \%$ halothane in oxygen, and triamcinolone was injected intrathecally slowly within $4 \mathrm{~s}$ with a microsyringe in the following concentrations: $0.3 \mathrm{mg} / \mathrm{kg}, 0.6 \mathrm{mg} / \mathrm{kg}$ once $(n=5 /$ group $)$ on day 11 post-immunization (p.i.) in a volume of $5 \mu \mathrm{l}$, as control $\mathrm{NaCl} 0.9 \%$ in a volume of $5 \mu \mathrm{l}$ was used $(n=5)$. The injection was performed with a $30-\mathrm{G}$ needle into the four to five lumbar intervertebral space, and the correct placement of the injection was confirmed by a movement of the tail ("tail flick") as described by Fairbanks and colleagues [17]. The experiments were repeated twice. 
The dosages described above were calculated according to previous experiments from Goericke et al. who showed a reduction of the volume of infarction after an injection of $0.0012-0.3 \mathrm{mg} / \mathrm{kg}$ TRIAM in the cisterna magna in a rat model of cerebral infarction [15].

\section{Electrophysiological analysis}

Nerve conduction tests were performed by a blinded investigator on the day before immunization $(-1)$ and on day 18 (maximum of natural disease course) p.i. The rats were anesthetized intraperitoneally (i.p.) with xylazine and ketamine $(10 \mathrm{mg} / \mathrm{kg}$ and $50 \mathrm{mg} / \mathrm{kg}$ respectively). Using a fully digital recording Keypoint apparatus (Dantec, Skovlunde, Denmark) and paired needle electrodes inserted into the sciatic notch (hip, proximal) or the popliteal fossa (distal), the sciatic nerve was stimulated with supramaximal rectangular pulses of $0.05 \mathrm{~ms}$ duration and the resulting compound muscle action potential (CMAP) was recorded from needle electrodes placed subcutaneously over the dorsal foot muscles. A ground electrode was placed between the distal stimulating electrode and the active recording electrode. To calculate the motor nerve conduction velocity (MNCV), the distance between stimulating cathodes was divided by the difference of the latency. Similarly, the persistence and minimum latency of $10 \mathrm{~F}$-waves evoked by stimulation at the popliteal fossa were recorded for the right side $[18,19]$. Temperature differences were minimized by conducting the study as soon as the anesthesia had taken effect and by warming the leg with a heating lamp.

\section{Schwann cell culture and immunocytochemistry}

Sciatic nerves from adult male Sprague Dawley rats (4 weeks old) were used as the source for Schwann cells (SCs), which were isolated and purified using established protocols [20]. For each culture, five animals were sacrificed by decapitation, their sciatic nerves collected and placed in Leibovitz's L-15 medium enriched with $50 \mu \mathrm{g} / \mathrm{ml}$ Gentamycin (Invitrogen). Nerves were stripped of epineurium and sectioned into 1-2 $\mathrm{mm}$ pieces. Explants were dissociated for $18 \mathrm{~h}\left(37^{\circ} \mathrm{C}, 5 \% \mathrm{CO}_{2}\right)$ with $1.25 \mathrm{U} / \mathrm{ml}$ dispase II (0.25\%) (Boehringer Mannheim Biochemicals) and $0.05 \%$ type I collagenase (Sigma) in DMEM with $50 \mu \mathrm{g} / \mathrm{ml}$ Gentamycin. Dissociation was stopped with HBSS containing 40\% fetal bovine serum (FBS) (Sigma), suspended and filtered through a $100-\mu \mathrm{m}$ strainer. SC cultures were expanded overnight on poly-L-lysine (Sigma) and $1 \mu \mathrm{g} / \mathrm{cm}^{2}$ laminin (Sigma) coated dishes in D-10 media (Dulbecco's modified Eagle's medium (DMEM) (Sigma) with $10 \%$ FBS) containing $50 \mu \mathrm{g} / \mathrm{ml}$ Gentamycin. For a rapid expansion of the SC population, the culture medium was supplemented with a combination of $10 \mathrm{nM}$ neuregulin (PeproTech) and $2 \mu \mathrm{M}$ forskolin (Sigma) as early as 1 day after plating. To maintain a low rate of fibroblast contamination, we used Miltenyi's MACS technology to achieve efficient separation of SCs and Thy-1-positive fibroblasts (negative selection) as described by the manufacturer's protocol. Then, the purified SCs (purification of 90\% SOX10-positive SC in flow cytometric analyses and immunocytochemistry) were kept in culture for further experiments (FACS analyses and RT-PCR analyses).

For immunocytochemical analyses, Schwann cells were seeded and incubated on poly-L-lysine- and laminin-coated coverslips for 2 days. After fixation with 4\% PFA, permeabilization with $0.1 \%$ PBS-Triton, and blocking with $10 \%$ normal serum, the cells were exposed to the primary antibody S-100 (Merck, MAB079-1, mouse-anti-rat, 1:500) or SOX10 (Abcam, ab155279, rabbit-anti-rat, 1:1000). Immunoreaction was detected with the secondary antibody, goat-anti-mouse IgG-Alexa Flour 488 (1:1000, Thermo Fisher Scientific), or goat-anti-rabbit IgG-Alexa Flour 488 (1:1000, Thermo Fisher Scientific), respectively. Furthermore, nuclei were counterstained with DAPI $\left(4^{\prime}, 6^{\prime}\right.$-diamidino-2-phenylindole. $2 \mathrm{HCl}$, Biozol, Eching, Germany). The omission of the primary antibodies served as negative control. Specificity of the staining was also controlled on sections of fibroblasts.

\section{Histopathological assessment and immunohistochemistry} After transcardial perfusion with PBS (Gibco) on day 18 p.i. (and day 23 p.i. for a FluoroMyelin staining) we dissected the two sciatic nerves, embedded their segments in Neg-50 (Thermo Fisher, Schwerte, Germany) and snap-frozen in liquid nitrogen. We used sections of rat tissue $(8 \mu \mathrm{m})$ on a cryostat (Leica Biosystems) mounted on glass slides (Hartenstein, Würzburg, Germany) for histopathology assessment.

For immunohistochemical staining, we used tissue sectioned in the cryostat and fixated in acetone at $-20{ }^{\circ} \mathrm{C}$ for $10 \mathrm{~min}$ and then exposed to the mouse monoclonal antibodies (mAb) anti-rat 15-6A1 (Hycultec, Pan T Cells CD3 1:100) and anti-rat ED1 (Hycultec, anti-CD68, macrophages, 1:100) using the avidin-biotin technique (Dako ARK KIT for Mouse Primary AB). We omitted the primary antibodies on the negative controls. Peripheral lymphoid organs served as a control for the specificity of the staining. Using 12 sections per animal and $\mathrm{a} \times 40$ magnification, we counted the number of positive cells. We present the results as the average cells per square millimeter tissue section.

For immunofluorescence, we incubated the sections after fixation with monoclonal antibodies (mAb) anti-ICAM-1 (1:100, Abcam, ab127160) and neurofilament $\mathrm{H}$ (clone N52, Abcam, 1:200), followed by incubation with secondary antibodies conjugated with Alexa 555 (1:1000) or Alexa 488 (1:1000) (Thermo Fisher, Schwerte, Germany) used according to the manufacturer's protocol. 
We imbedded the slides with Fluoromount containing 4',6' -diamidino-2-phenylindole.2HCl (DAPI) (Biozol, Eching, Germany) for fluorescent staining of DNA. For picturing the tissue, we used an inverted fluorescence microscope (BX51; Olympus, Tokyo, Japan) equipped with an Olympus DP50 digital camera.

We identified the demyelination through the accumulation of nuclei and absence of FluoroMyelin ${ }^{\text {Tx }}$ Red fluorescent stain (1:300, Invitrogen, Germany) performed according to the manufacturer's protocol. For the statistic, we used the images ( $\times 20$ magnification) of 12 transverse sections of the sciatic nerve from each animal (Cell F 5.1, Olympus, Tokyo, Japan) and determined the percentage of the area of demyelination per section using image analysis software (ImageJ, National Institutes of Health, Bethesda, USA).

For histopathological assessment and immunohistochemistry, slides were blinded by a not-involved third person and labeled with a numeric code, which was unblinded after analysis.

\section{Isolation of mononuclear cells from lymph nodes and FACS analyses}

The inguinal lymph nodes were removed after transcardial perfusion with PBS (Gibco) on day 18 p.i. under aseptic conditions. Single cell suspensions of mononuclear cells from individual rats were prepared separately ( $n=5 /$ group). We evaluated the frequency of $C D 4^{+}$ $\mathrm{T}$ cells, $\mathrm{CD}_{11 \mathrm{~b}^{+}}$cells, $\mathrm{CD} 4^{+} \mathrm{CD} 11 \mathrm{~b}^{+}$dendritic cells (DCs), and $\mathrm{CD}^{+} \mathrm{CD}^{+} 5^{+} \mathrm{FoxP3}^{+}$regulatory $\mathrm{T}$ cells (Tregs) by fluorescence-activated cell sorting (FACS) staining (eBioscience, San Diego, CA). FACS analyses were performed with a FACS Canto II (BD Pharmingen, Heidelberg, Germany) machine and FlowJo software (Tree Star, Ashland, Oregon). Monoclonal antibodies purchased from BD Pharmingen or eBioscience were used to detect CD4-FITC (1:500), CD11b-PE (1:200), CD25-APC (1:100), and MHC-II-Alexa647 (1:300) in accordance with the manufacturer's instructions. Intracellular staining for Foxp3 was performed using the Foxp3 Staining Set (eBioscience) according to the manufacturer's instructions.

To examine the protective role of TRIAM against oxidative stress, we incubated SCs for $24 \mathrm{~h}$ with $0.03 \mathrm{mM}$ $\mathrm{H}_{2} \mathrm{O}_{2}$ with and without $10 \mu \mathrm{g} / \mathrm{ml}$ TRIAM. Cell survival was analyzed with flow cytometry (propidium iodide staining, Thermo Fisher, as described by the manufacturer's protocol). The TRIAM concentration was chosen after titration experiments with propidium iodide (PI) staining to exclude toxicity. The $\mathrm{H}_{2} \mathrm{O}_{2}$ concentration was chosen after titration experiments with PI staining to generate a sub-maximal lethality rate. We were able to generate a sub-maximal MHCII expression after incubation with $100 \mathrm{U} / \mathrm{ml}$ IFN gamma [21]. To examine the
MHCII expression after INF gamma stimulation with and without $10 \mu \mathrm{g} / \mathrm{ml}$ TRIAM treatment, we stained with MHCII Alexa 647 antibody and analyzed with flow cytometric analyses as described above.

\section{Tissue preparation, RNA isolation, and gene expression analyses with quantitative RT-PCR}

Total RNA was isolated from the sciatic nerve samples of rats at disease maximum, 18 days p.i. using the RNeasy Mini extraction kit (Qiagen, Hilden, Germany). All samples were treated with the RNA Stabilization Reagent (RNAlater, Qiagen, Hilden, Germany) at $37^{\circ} \mathrm{C}$ overnight and stored at $-80^{\circ} \mathrm{C}$ until use. Total RNA was reverse-transcribed into cDNA according to the manufacturer's protocol for the Reverse Transcription System (Promega, Madison, WI, USA).

Sequence-specific primers were designed with the following, sen (sense), ase (anti-sense), then mRNA expression levels were analyzed by quantitative RT-PCR according to the manufacturer's instructions (Applied Biosystems, Foster City, CA, USA):

FoxP3 (sen AGG CAG AGG ACA CTC AAT GAA, ase ACT GCT CCC TTC TCA CTC TCC), IFN gamma (sen AAA GAC AAC CAG GCC ATC AG, ase CTT TTC CGC TTC CTT AGG CT), IL-10 (sen CCT GCT CTT ACT GGC TGG AG, ase TCT CCC AGG GAA TTC AAA TG), and IL-4 (sen TGA TGG GTC TCA GCC CCC ACC TTG C, ase CTT TCA GTG TTG TGA GCG TGG ACT C).

RT-PCR amplifications were carried out using the RT-PCR System 7500 (Applied Biosystems) via protocol described by Pfaffl et al. [22]. In this context, the relative expression ratio is calculated only from the RT-PCR efficiencies and the crossing point deviation of the sample versus a control. $\beta$-Actin and GAPDH were used to normalize relative mRNA expression. Each experiment was performed in duplicate, and the mean $\mathrm{Ct}$ was used in the equation for the housekeeping genes and $\mathrm{Ct}$ for the genes of interest.

Total mRNA was isolated from Schwann cells, and RT-PCR analyses were performed for TLR-4 (toll-like receptor-4, sense GCGCCTAAAACCCATTATGTT, anti-sense TGATTCTTTGCCTGAGTTGCT) and IL-1Ra (IL-1 receptor antagonist, sense GTGTGATGC CCCTAAACTGAA, anti-sense AACCTCTTTAGGCA GCTCTGG) as described above.

\section{Statistical methods}

Statistical analyses were performed by GraphPad Prism 7 software (GraphPad Software Inc., San Diego, USA). Data are provided as mean \pm SEM (standard error of mean) for the clinical score or as mean \pm SD (standard deviation) for the rest of the statistical analyses. Differences between pairs of groups were tested by Student's $t$ 
test. Differences between three or more groups were tested by one-factor analysis of variance (ANOVA). The area under the curve (AUC) was calculated for clinical courses and analyzed by one-way analysis of variance (ANOVA) combined with Tukey's multiple comparison test. The chi-square test was used to analyze categorical outcomes (disease incidence). Probability level ( $p$ value) are indicated as $* p \leq 0.05,{ }^{* * *} p \leq 0.01,{ }^{* * * *} p \leq 0.001$, and ***:*** $p \leq 0.0001$.

\section{Results}

Intrathecal triamcinolone applied in a therapeutic concept ameliorates the clinical course of experimental autoimmune neuritis

After immunization with P2 protein peptide 53-78, clinical signs of EAN begun around day 10 p.i. At day 11 p. i., $0.9 \% \mathrm{NaCl}$ or TRIAM were applied intrathecally. The incidence of EAN for the control group was $100 \%$, and the groups receiving TRIAM showed an incidence of $50 \%$ $(0.3 \mathrm{mg} / \mathrm{kg})$ and $20 \%(0.6 \mathrm{mg} / \mathrm{kg})$ respectively $\left({ }^{* * *} p<0.01\right)$. A remarkable dose-dependent amelioration of EAN clinical signs was documented (AUC, NaCl-treated vs. 0.6 $\mathrm{mg} / \mathrm{kg}$ TRIAM $* * 00.001, n=5 /$ group) (Fig. 1a, b).

\section{Intrathecal triamcinolone improved proximal and distal nerve conduction}

To elucidate the effect of intrathecal TRIAM regarding proximal and distal demyelination, we performed electrophysiological measurements of the sciatic nerve at the maximum of the clinical course (day 18 p.i.) as described in the "Materials and methods" section.

The average minimum latencies of the elicitable F-waves were slightly prolonged in $\mathrm{NaCl}$-treated group at day 18 p.i. compared to day -1 p.i., whereas there was no difference for the 0.3 and $0.6 \mathrm{mg} / \mathrm{kg}$ TRIAM-treated groups (average minimum $\mathrm{F}$-wave latencies of the control group on day -1 p.i. $8.2 \mathrm{~ms}$ vs. day 18 p.i. $14.2 \mathrm{~ms}$, $" p<0.05, n=5$, experiment repeated twice).

$\mathrm{MNCV}$ was significantly reduced in the $\mathrm{NaCl}$-treated rats (mean $\mathrm{MNCV}$ on day 18 p.i. $35.6 \mathrm{~m} / \mathrm{s}$ vs. day -1 p.i. $56.9 \mathrm{~m} / \mathrm{s}, \quad * * * 0.0001, n=5$, experiment repeated twice), whereas no difference of the mean MNCV on day -1 was seen for TRIAM-treated groups (Fig. 2), indicating the additional protective role of intrathecal application of TRIAM against distal demyelination.

\section{Intrathecal triamcinolone reduces histological signs of demyelination and $\mathrm{T}$ cell and macrophage infiltration in the sciatic nerves}

Figure 3a shows representative pictures of FluoroMye$\operatorname{lin}^{\text {tw }}$ staining. Administration of 0.3 and $0.6 \mathrm{mg} / \mathrm{kg}$ intrathecal TRIAM significantly reduced the percentage of demyelination of the sciatic nerves when compared to $\mathrm{NaCl}$-treated rats on day 23 p.i. ${ }^{* * * *} p<0.0001, n=5$
Fig. 3b, experiment repeated once) but not on day 18 p.i. (experiment repeated twice, data not shown).

Next, we showed that clinical improvement correlates with the reduction of inflammatory infiltration of the PNS. Histological data demonstrating inflammation within the PNS are depicted in Fig. 4. Intrathecal administration of 0.3 and $0.6 \mathrm{mg} / \mathrm{kg}$ TRIAM on day $11 \mathrm{p}$.i. significantly reduced infiltration of macrophages and lymphocytes in the sciatic nerves compared to the $\mathrm{NaCl}$-treated control group, when examined on day 18 p.i. ${ }^{* * *} p<0.0001$, Fig. $\left.4 \mathrm{a}, \mathrm{b}\right)$. Interestingly, inflammatory infiltrates were reduced both in the distal and proximal parts of the sciatic nerves (data not shown).

Furthermore, ICAM-1 expression was significantly reduced for 0.3 and $0.6 \mathrm{mg} / \mathrm{kg}$-treated rats correlating with the prominent reduction of inflammatory infiltrates for these concentrations ${ }^{* * * *} p<0.0001$, Fig. $\left.4 \mathrm{c}\right)$. Axonal staining with neurofilament $\mathrm{H}$ antibody showed less axonal damage for the low TRIAM dosage (" $p<0.05$, Fig. $4 \mathrm{~d}$ ).

\section{Intrathecal triamcinolone induced a Th2 cytokine shift in the sciatic nerve at disease maximum as well as a reduction of peripheral lymphocytes}

Furthermore, a Th2 cytokine shift was observed for both concentrations of 0.3 and $0.6 \mathrm{mg} / \mathrm{kg}$ TRIAM at day 18 p.i., as IL-4 mRNA expression showed a statistically significant increase in contrast to TNF $\alpha$ and IFN- $\gamma$, which decreased at the disease maximum. IL-10 however was also reduced at that time point (Fig. 5, ${ }^{* * * *} p<0.0001, n=5$ /group, experiment repeated twice).

To detect possible effects on the secondary lymphoid organs, we also isolated peripheral mononuclear cells from the inguinal lymph nodes and spleen at day 18 p.i. and analyzed them with flow cytometry. We evaluated the frequency of $\mathrm{CD}^{+} \mathrm{T}$ cells, CD $11 \mathrm{~b}^{+}$cells, $\mathrm{CD} 4^{+}$ $\mathrm{CD}_{11 \mathrm{~b}^{+}}$dendritic cells (DCs) and CD4+ CD25+ FoxP3+ regulatory $\mathrm{T}$ cells (Tregs). Apart from a reduction of the percentage of lymphocytes in spleen (lymphocytes in the control group; mean $\pm \mathrm{SD}$ of $15.8 \pm 4.4 \%, \quad 0.3 \mathrm{mg} / \mathrm{kg}$ TRIAM $3.4 \pm 0.3 \%, 0.6 \mathrm{mg} / \mathrm{kg}$ TRIAM $5.5 \pm 1.6 \%$ of all cells, control group vs. $0.3 \mathrm{mg} / \mathrm{kg}$ group, ${ }^{* * *} p<0.001$ ) no further statistically significant differences were found between TRIAM and $\mathrm{NaCl}$-treated rats for all populations tested.

\section{Anti-inflammatory and antioxidative effects of triamcinolone on Schwann cell cultures}

Representative pictures of immunohistochemical staining of SC culture with SOX10 and DAPI are presented in Fig. 6 .

After incubation with $10 \mu \mathrm{g} / \mathrm{ml}$ TRIAM for $24 \mathrm{~h}$, we were able to detect a significant reduction in the expression of the inflammatory TLR-4 as well as an increase in the anti-inflammatory cytokine IL-1Ra by RT-PCR (Fig. 6 b, c). 

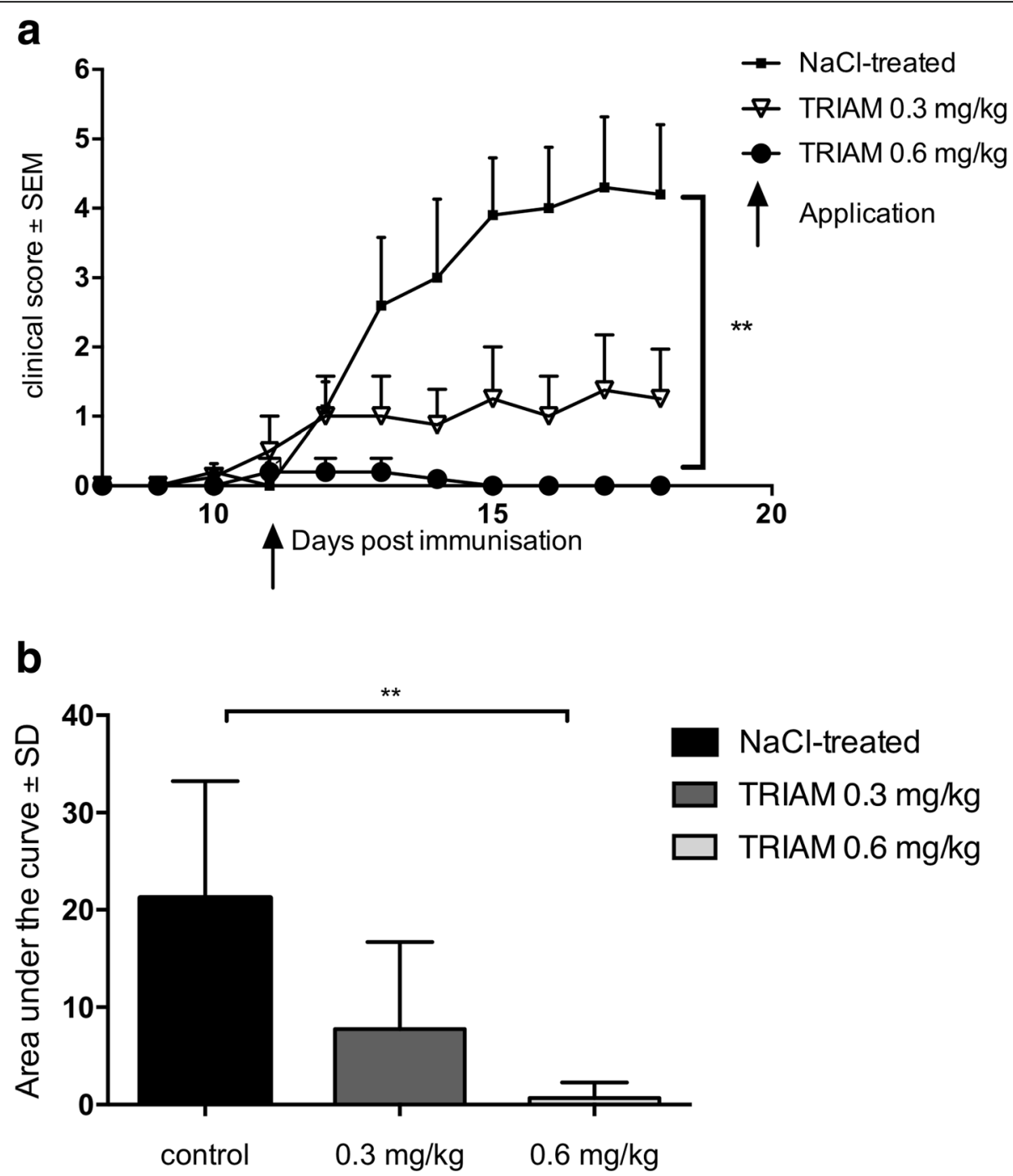

Fig. 1 Representative clinical course of EAN rats. a. EAN was induced in Lewis rats by immunization on day 0 with P253-78 peptide plus CFA. Rats received human triamcinolone (TRIAM) intrathecally at doses of $0.3 \mathrm{mg} / \mathrm{kg}$ and $0.6 \mathrm{mg} / \mathrm{kg}$ on day 11 p.i. Control rats received $\mathrm{NaCl} 0.9 \%$ only. The experiment was repeated two times, each time with $n=5 / \mathrm{group}$. Mean values and SEM are depicted. Statistical analysis and $p$ value calculation were performed calculating the area under the curve (AUC). $\mathbf{b}$ Comparison of AUC was done using one-way ANOVA combined with Tukey's multiple comparisons test. Mean values and SD are depicted (NaCl-treated vs $0.6 \mathrm{mg} / \mathrm{kg} ;{ }^{* *} p<0.001, n=5$ )

To induce oxidative stress, we incubated the SCs with $0.03 \mu \mathrm{M} \mathrm{H} \mathrm{H}_{2} \mathrm{O}_{2}$ for $24 \mathrm{~h}$. Co-incubation with $10 \mu \mathrm{g} / \mathrm{ml}$ TRIAM resulted in an increased cell viability in PI staining (Fig. 6d).

To induce MHCII expression, we incubated SCs for 5 days with $100 \mathrm{U} / \mathrm{ml}$ interferon- $\gamma$. Interestingly, after co-incubation with TRIAM, MHCII expression was significantly increased. A potentially toxic effect of the substances used in the concentrations indicated was excluded by means of PI staining (Fig. 6e).

\section{Discussion}

In our current study, we present a new perspective of the classical treatment of autoimmune neuropathies using the route of intrathecal application of TRIAM. Thereby, we introduce two novel aspects of the action of intrathecal TRIAM: an anti-inflammatory potential for autoimmune neuritis in vivo and a direct immunomodulatory/antioxidative effect on Schwann cells in vitro.

Intrathecal TRIAM has been established as an off-label treatment to improve spasticity and walking distance in patients with chronic forms of multiple sclerosis. Patients with spinal symptoms respond particularly well to this application route for up to 3 months after application. Due to the dose sparing effect, TRIAM therapy is rarely accompanied by long-term side effects, such as osteoporosis and weight gain, which are usually observed during repeated pulsed intravenous steroid infusions [4]. 


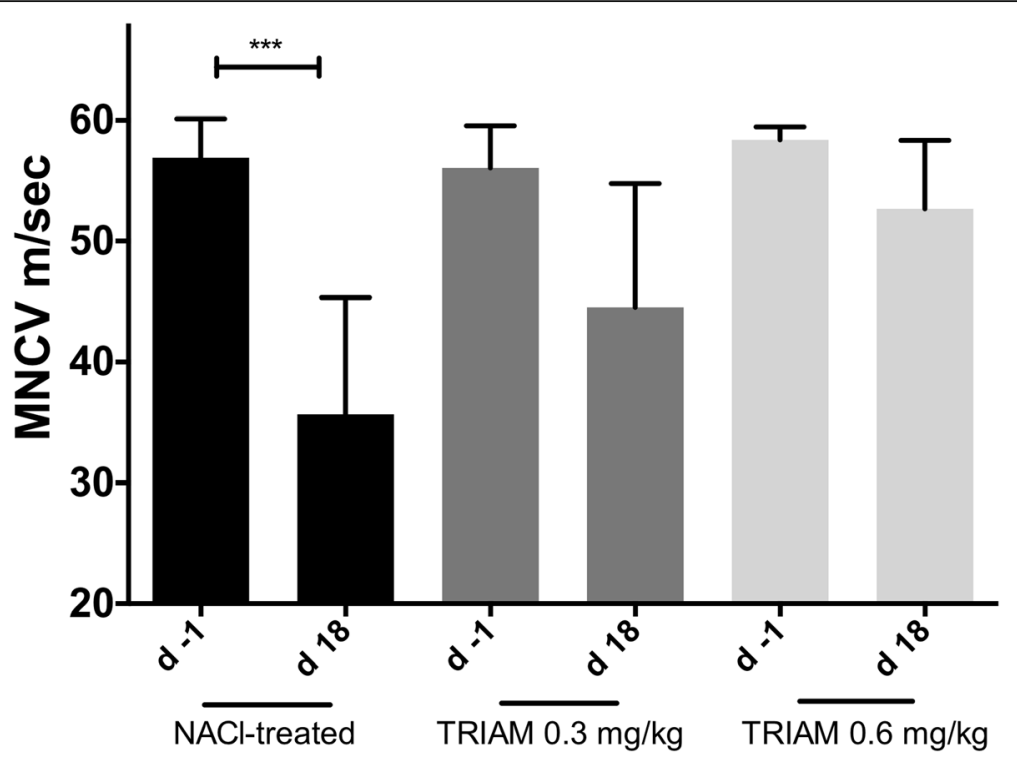

Fig. 2 Motor nerve conduction velocity analyses of the sciatic nerve. Motor nerve conduction velocity of the sciatic nerve 1 day before immunization (d -1) and at the peak of disease (day 18 p.i.) ( $n=5 /$ group): After proximal and distal stimulation of the sciatic nerve, the conduction velocity (MNCV) was calculated. A statistically significant reduction of the MNCV appeared only for the NaCl-treated group $(n=5)$ but no difference in the MNCV was seen for all triamcinolone (TRIAM)-treated groups. Mean values and SD are depicted; $p$ values, ${ }^{* *} p<0.0001$. The experiments were repeated two times with $n=5 /$ group
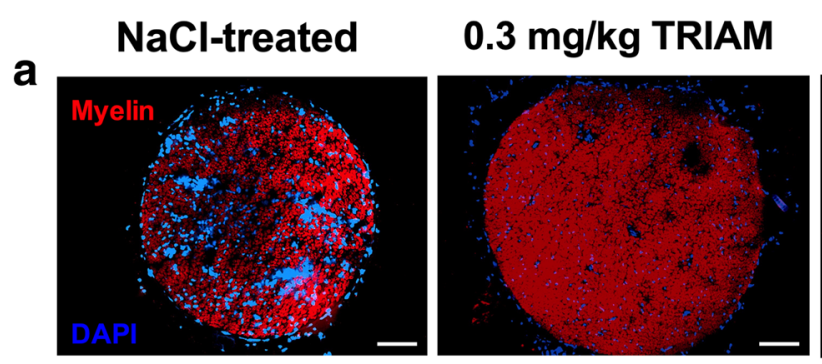

\section{$0.6 \mathrm{mg} / \mathrm{kg}$ TRIAM}

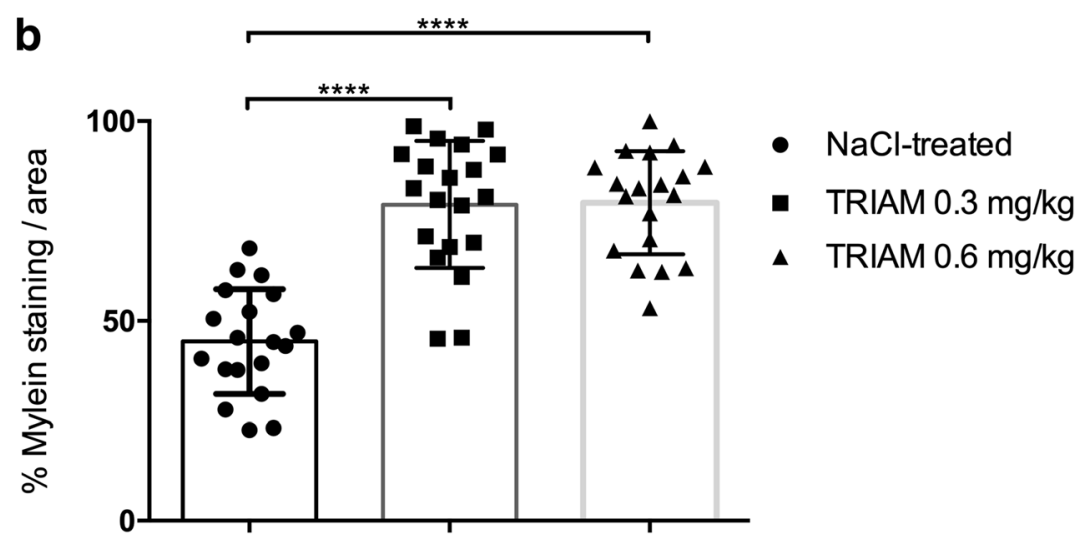

Fig. 3 FluoroMyelin staining of the sciatic nerve. a Representative pictures and statistical analysis of FluoroMyelin staining for sciatic nerve transverse sections of rats ( $n=5 /$ group) treated with triamcinolone (TRIAM) 0.3 and $0.6 \mathrm{mg} / \mathrm{kg}$ and NaCl-treated animals, showing massive demyelination for $\mathrm{NaCl}$-treated rats and preservation of myelin for triamcinolone-treated groups (b, mean values and SD are depicted, $\left.{ }^{* * *} p<0.0001\right)$ at day 23 p.i. No effect on demyelination was found on day 18 p.i. (experiments repeated twice). The experiment was performed once for day 23 p.i. Scale bars indicate $100 \mu \mathrm{m}$ 


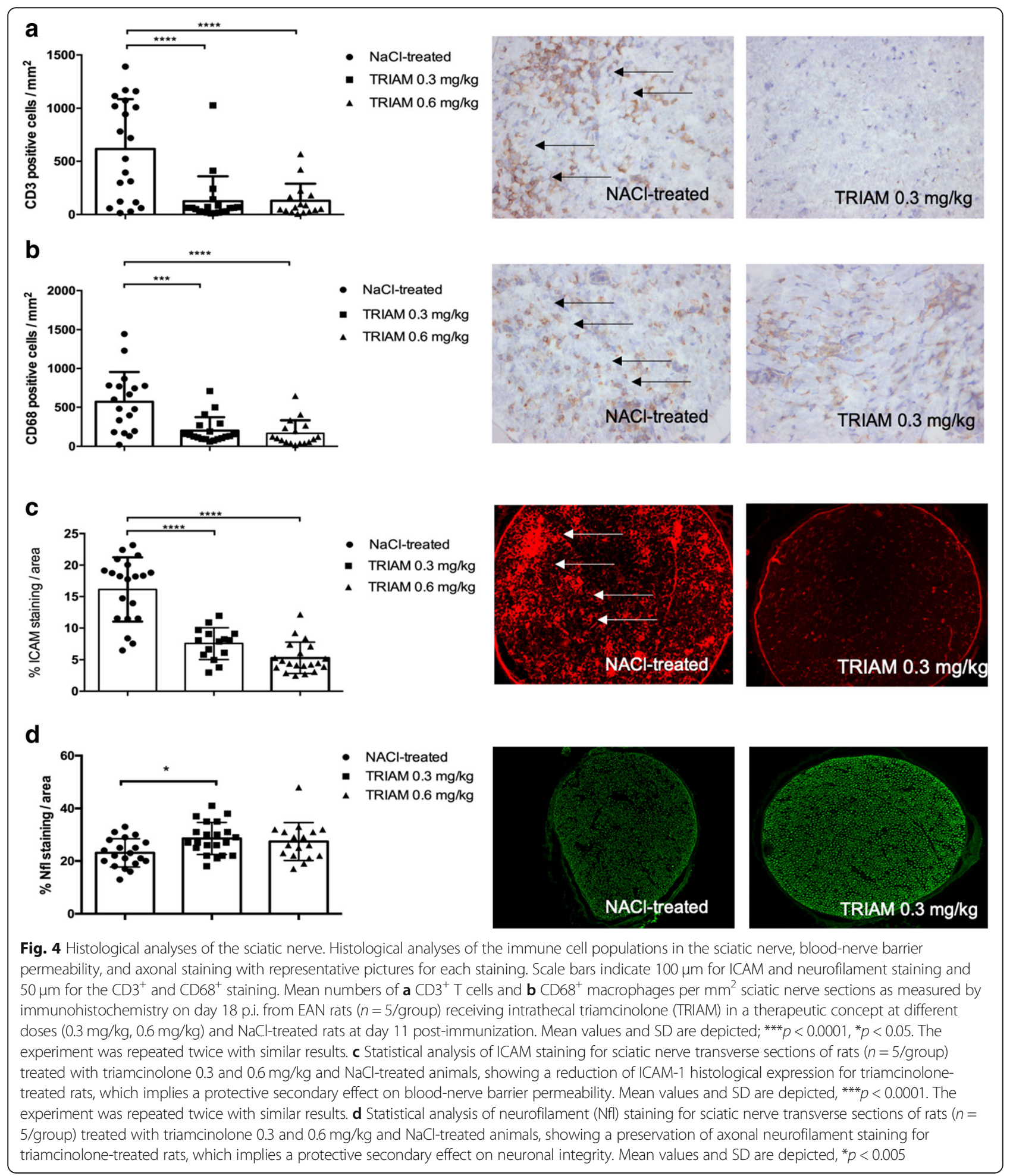

The mode of action of TRIAM is not completely understood since the scientific evidence of its efficacy relies on clinical observations and not on placebo-controlled trials. According to studies on MS patients, a potential effect of intrathecal TRIAM characterized in CSF is the reduction of repulsive guidance molecule A (RGMa), a cell death regulator [23]. Recurrent TRIAM applications induced a decreased concentration of RGMa fragments combined with a decline in free radical concentration, potentially improving neuronal regeneration $[6,7]$. 


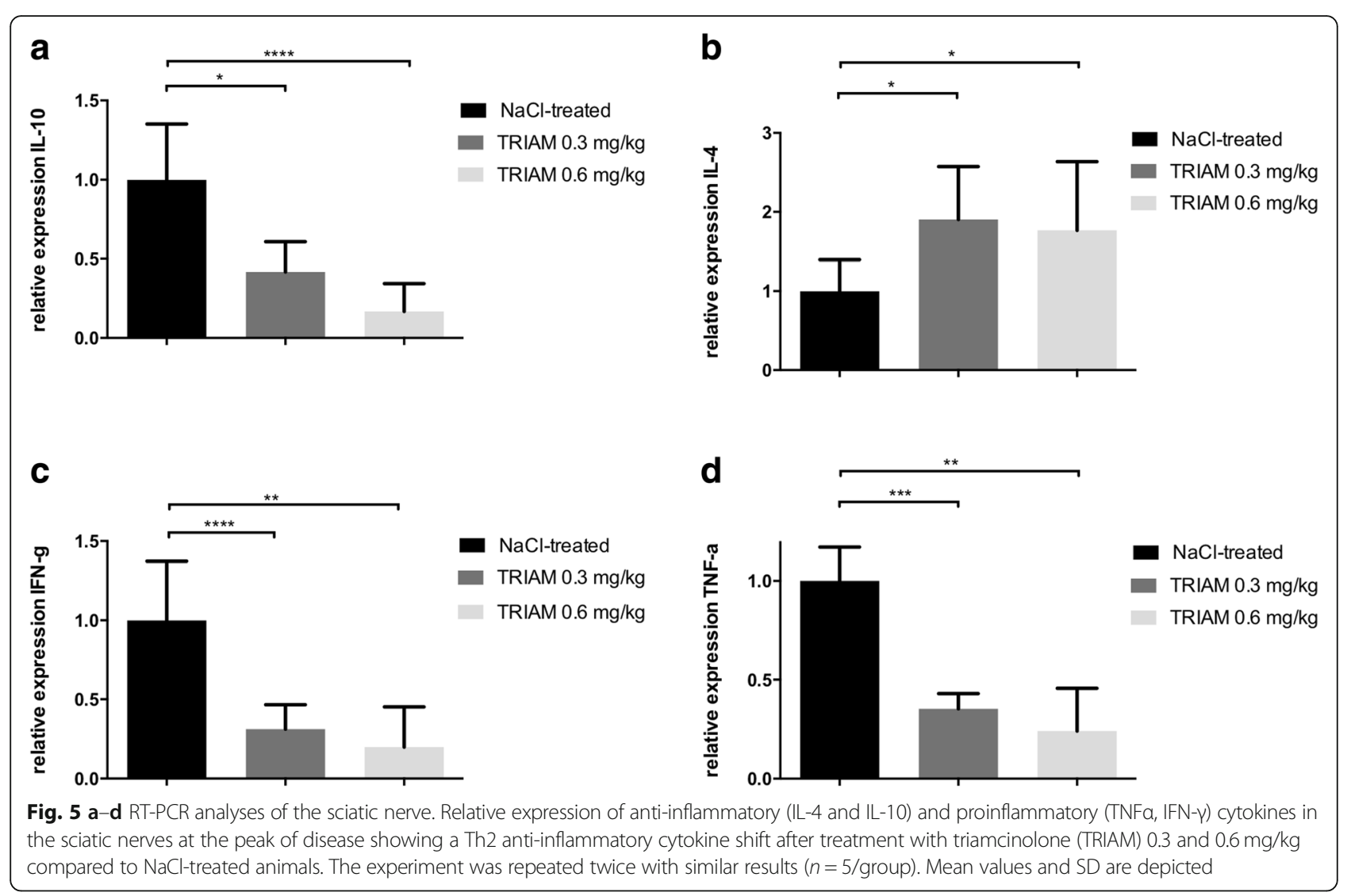

On the other hand, there is only one study suggesting that TRIAM could be effective in the treatment of peripheral neuropathies and most specifically on the treatment of neuropathic pain. TRIAM was applied subcutaneously 5 days after a nerve injury to rats with an experimental post-traumatic painful peripheral neuropathy. TRIAM-treated rats had a statistically significant reduction in the magnitude of heat-hyperalgesia and mechano-allodynia. The proposed mechanism of action was a reduction of TNF $\alpha$ in endoneurial mast cells [24].

However, the mode of action of the intrathecal application has not been investigated for autoimmune diseases of the PNS despite proximal route involvement and early intrathecal protein increase.

We show for the first time, that in a therapeutic concept, TRIAM achieved a dose-dependent improvement of clinical signs, in combination with a remarkable reduction of inflammatory infiltrates in the sciatic nerves. Nerve conduction studies showed a prominent preservation of $\mathrm{F}$-waves as an indicator of an improvement of proximal demyelination as well as an unchanged motor conduction velocity as a sign of a distal preservation of myelination. Demyelination reduction was more pronounced at day 23 p.i. and not at day 18 p.i. (maximum of disease) implying a late effect of the reduction of the inflammatory infiltrations on demyelination or a late improvement of remyelination. Therefore, we conclude that intrathecal TRIAM injection in the effector phase of autoimmune neuritis reduces inflammatory activity beginning at the injection sites and extending into the whole nerve thereby achieving enough improvement of electrophysiological signs of demyelination.

To define possible mechanisms of action of the local intrathecal injection of TRIAM, we analyzed possible immunomodulatory mechanisms in the PNS reported before for systemically applied corticosteroids.

Firstly, the reduction of inflammatory infiltrates correlated with the downregulation in the histological expression of ICAM molecule in the peripheral nerves. This effect has been reported before for patients with chronic inflammatory demyelinating neuropathy and is probably secondary to the reduction of mononuclear cell infiltration [25]. This observation is crucial and could be easily evaluated in patients with CIDP receiving repeated intrathecal TRIAM applications, through a reduction of total protein in the CSF, which is the marker of blood-nerve barrier permeability. Neurofilament staining showed a reduction of axonal loss after TRIAM treatment probably as a secondary effect to the impressive anti-inflammatory effect of TRIAM.

Furthermore, the anti-inflammatory effects on cell infiltrates correlated with a Th2-cytokine (anti-inflammatory) 


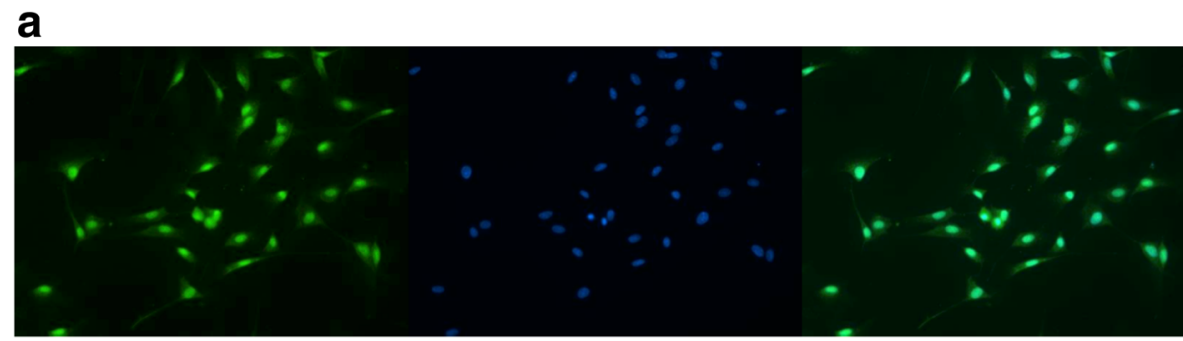

Merge

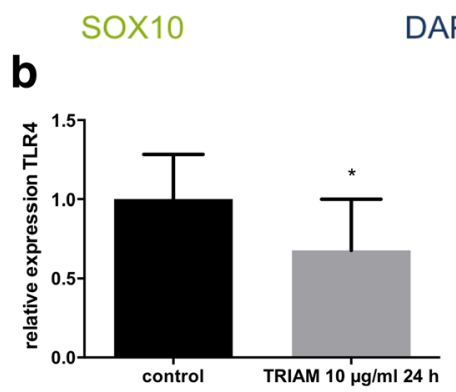

b

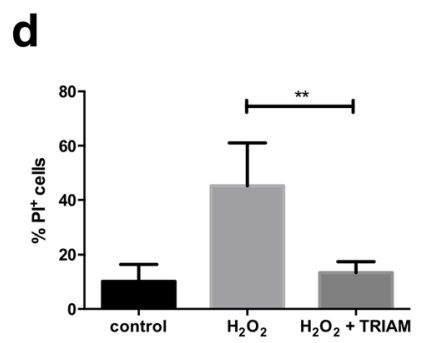

C

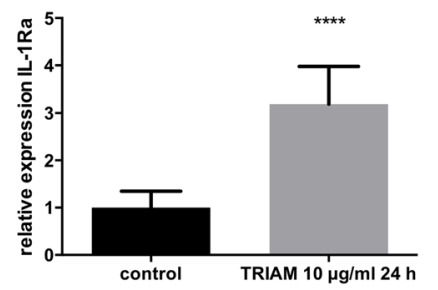

e

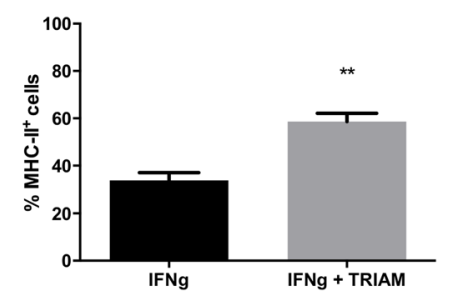

Fig. 6 In vitro analyses of Schwann cells culture. a Immunohistochemical staining of Schwann cell culture with SOX10 and DAPI (nuclear staining). b, c After incubation of Schwann cells with $10 \mu \mathrm{g} / \mathrm{ml}$ triamcinolone (TRIAM) for $24 \mathrm{~h}$, we detected a significant reduction in the relative gene expression of the pro-inflammatory TLR-4 as well as an increase in the anti-inflammatory cytokine IL-1Ra by RT-PCR. Mean values and SD are depicted. d Incubation of Schwann cells for $24 \mathrm{~h}$ with $0.03 \mathrm{mM} \mathrm{H}_{2} \mathrm{O}_{2}$. Co-incubation with $10 \mu \mathrm{g} / \mathrm{ml}$ triamcinolone resulted in increased cell viability in propidium iodide (PI) staining. Mean values and SD are depicted. e Incubation of Schwann cells with $100 \mathrm{U} / \mathrm{ml}$ interferon- $\gamma$. After co-incubation with triamcinolone, MHC II expression in flow cytometric analyses was significantly increased. Mean values and SD are depicted. ${ }^{*} p \leq 0.05,{ }^{* *} p \leq 0.01,{ }^{* *} p \leq 0.001$, and ${ }^{* * *} p \leq 0.0001$

shift (increase of IL- 4 and decrease of IFN- $\gamma$ and TNF $\alpha$ ) in the peripheral nerves, confirming the effects of TRIAM in the gene expression level. Our findings are crucial as previous experiments have shown that activated glucocorticoid-receptor complex can bind to and inactivate key proinflammatory transcription factors (e.g., NF kappa B). While steroid treatment broadly attenuates cytokine production, it cannot modulate it selectively, e.g., just the Th0, the Th1, or the Th2 pathways. The production of the "anti-inflammatory" IL-10 is also inhibited, as shown in our experiments [26]. However, through an increase of IL-4, a Th2 cytokine shift is still achieved in our model, which implies an effective anti-inflammatory action.

We next considered the potential site of action of intrathecal TRIAM in the PNS, focusing on SCs, as the major cell population initially affected during demyelinating neuropathies. Previous in vitro studies reveal that SCs express glucocorticoid receptors whereas GR is expressed in the nuclei of SCs in vivo in intact and injured sciatic nerves. Glucocorticoids (hydrocortisone, dexamethasone) enhance the potency of SC proliferation $[27,28]$. Furthermore, they enhance the rate of myelin formation [29] and stimulate the activity of promoters of peripheral myelin protein-22 (PMP22) and myelin protein zero $(\mathrm{PO})$ genes $[30,31]$.

However, the in vitro effects of TRIAM on SCs have not been investigated before. Our data imply a combined anti-inflammatory and anti-oxidative effect of TRIAM on SCs.

Lipopolysaccharide-induced inflammatory cytokine production by SCs is dependent upon TLR4 expression. In vitro data showed that TLR4 expression is upregulated after sciatic nerve injury of rat and modulation of its expression increased relative gene expression of proinflammatory molecules such as c-Jun and extracellular signal-regulated kinase (ERK) [32]. In our in vitro model, TRIAM reduced significantly TLR4 expression implying an anti-inflammatory potential of SCs after TRIAM treatment. 
Furthermore, IL-1Ra production was reduced after SC incubation with TRIAM. Experiments on sciatic nerves of EAN rats after immunization with P2 and after adoptive transfer of effector $\mathrm{T}$ cells have shown that in both models, IL-1a was expressed by SCs, during preclinical EAN whereas IL-1Ra was not detectable in SCs at this stage. However, clinically manifest EAN was characterized by SC-specific expression of IL-1Ra, mostly on the paranodal regions, sites essential for proper impulse transmission. These data indicate that SC-specific autoregulation of IL-1Ra is highly relevant for immune regulation at paranodes during autoimmune neuritis [33].

TRIAM also protected SCs from $\mathrm{H}_{2} \mathrm{O}_{2}$-mediated oxidative stress. Oxidative stress is the main characteristic of autoimmune inflammation and leads to neuronal degeneration, a crucial parameter of disability for patients with chronic immune neuropathies. Treatment options, which reduce oxidative stress and therefore improve neuronal survival, are therefore essential for these patients. Furthermore, in line with our experiments, CSF analyses in MS patients $(n=16)$ after one intrathecal TRIAM injection revealed an increase of $\mathrm{Cu}$ (II) ion absorption, which reflects an augmented content of reduced proteins and an alteration of the redox potential in cerebrospinal fluid, through a decline of reactive oxygen species [34].

SC showed however a pro-inflammatory phenotype after TRIAM and IFN- $\gamma$ incubation through the increase of MHCII expression. We interpret these results in the context of the crystalline structure of TRIAM, which could be presented as antigen on MHCII class molecules thereby initiating an immune response. This effect could explain cases of constrictive arachnoiditis and sterile meningitis reported after repeated intrathecal TRIAM injection [4]. However, we must point out that CSF investigations regarding markers for cell damage, such as neuron-specific enolase, S-100, neurofilament heavy chain, tau protein provided no convincing evidence of neuronal or SC damage after the intrathecal TRIAM treatment [35]. The interaction of substances applied intrathecally with the MHCII molecule and its role for sterile meningitis remains to be further investigated as other substances with non-crystalline structure can also lead to this side effect [36]. As we did not investigate the effects of TRIAM in neuron-SC cocultures regarding myelination, we can only postulate that the late effect on myelination shown on day 23 p.i. could be attributed on an improved SC myelination function.

\section{Conclusions}

In conclusion, we present a new perspective for corticosteroids treatment in peripheral neuropathies by applying intrathecal TRIAM in a therapeutic concept.
The major shortcoming of our in vivo study is the fact that the monophasic animal model of EAN does not reproduce the complexity of the pathophysiology of CIDP but mostly the one of GBS, for which at least intravenous corticosteroids are not effective. Therefore, we chose the therapeutic concept in order to investigate the effects of TRIAM in the effector phase (when clinical signs begin to appear), which can be recognized both in GBS and CIDP patients in everyday practice.

Since intrathecal TRIAM treatment has already been used for patients with multiple sclerosis, the transfer of our application model in human subjects with CIDP could be tested in clinical studies. As corticosteroids are already used for patients with CIDP in the case of sensory symptoms and painful neuropathic pain, a probable anti-inflammatory, anti-oxidative, and secondary neuroprotective beneficial effect of intrathecal TRIAM application as well the dose-sparing effect must be considered. In light of our current observation, intrathecal application of TRIAM could be a promising treatment option for neuritis patients.

\section{Abbreviations \\ AUC: Area under the curve; CFA: Complete Freund's adjuvant; CIDP: Chronic inflammatory demyelinating polyneuropathy; CMAP: Compound muscle potentials; DAPI: 4',6-Diamidino-2-phenylindole; EAN: Experimental autoimmune neuritis; GBS: Guillain-Barré syndrome; i.p.: Intraperitoneally; ICAM1: Intercellular adhesion molecule 1; MNCV: Motor nerve conduction velocity; p.i.: Post-immunization; PBS: Phosphate-buffered saline; PNS: Peripheral nervous system; TRIAM: Triamcinolone acetonide}

\section{Acknowledgements}

Not applicable

\section{Funding}

There was no funding source.

\section{Availability of data and materials}

The datasets used and/or analyzed during the current study are available from the corresponding author on reasonable request.

\section{Authors' contributions}

MS, HB, TG, JM, XP, participated in the acquisition of data, analysis and interpretation of data, and revising the manuscript for content. BA, MSY, and RG participated in the study design and drafted and revised the manuscript for content. All authors read and approved the final manuscript.

\section{Ethics approval and consent to participate}

All experiments were reviewed and approved by the North-Rhine-Westphalia, Germany authorities for animal experimentation (TVA 84-02.04.2017-A023).

\section{Consent for publication}

Not applicable

\section{Competing interests}

Kalliopi Pitarokoili received travel funding and speaker honoraria from Biogen Idec, Novartis and Bayer Schering Pharma and funding from the RuhrUniversity, Bochum (FORUM-Program). Thomas Grüter received a travel reimbursement from Sanofi Genzyme and Biogen Idec, none related to this manuscript. Jeremias Motte received travel grants from Biogen Idec, none related to this work. Björn Ambrosius received travel grants from Novartis, not related to this manuscript. R. Gold has received consultation fees and speaker honoraria from Bayer Schering, Biogen idec, Merck Serono, Novartis, Sanofi-Aventis, and TEVA. He also acknowledges grant support from Bayer Schering, Biogen idec, Merck Serono, Sanofi-Aventis, and TEVA, none related 
to this manuscript. Min-Suk Yoon has received speaker honoraria from CSL Behring and Grifols, scientific grant from CSL Behring, none related to this manuscript. The other authors declare that they have no competing interests.

\section{Publisher's Note}

Springer Nature remains neutral with regard to jurisdictional claims in published maps and institutional affiliations.

\section{Received: 13 September 2018 Accepted: 25 February 2019 Published online: 09 March 2019}

\section{References}

1. Yang Y, Bailey C, Loewenstein A, Massin P. Intravitreal corticosteroids in diabetic macular edema: pharmacokinetic considerations. Retina. 2015; 35(12):2440-9.

2. Madalena KM, Lerch JK. The effect of glucocorticoid and glucocorticoid receptor interactions on brain, spinal cord, and glial cell plasticity. Neural Plast. 2017;2017:8640970

3. Hellwig K, Stein FJ, Przuntek H, Müller T. Efficacy of repeated intrathecal triamcinolone acetonide application in progressive multiple sclerosis patients with spinal symptoms. BMC Neurol. 2004;4(1):18.

4. Müller T. Role of intraspinal steroid application in patients with multiple sclerosis. Expert Rev Neurother. 2009;9(9):1279-87.

5. Hoffmann V, Kuhn W, Schimrigk S, Islamova S, Hellwig K, Lukas C, Brune N, Pöhlau D, Przuntek H, Müller T. Repeat intrathecal triamcinolone acetonide application is beneficial in progressive MS patients. Eur J Neurol. 2006: 13(1):72-6.

6. Müller T, Herrling T, Lütge $S$, Küchler M, Lohse L, Rothe H, Haas T, Marg M, Öhm G, Jung K. Reduction in the free radical status and clinical benefit of repeated intrathecal triamcinolone acetonide application in patients with progressive multiple sclerosis. Clin Neuropharmacol. 2014;37(1):22-5.

7. Müller T, Lütge S. Biochemical indicators for neuronal regeneration during intrathecal triamcinolone application in multiple sclerosis. Neural Regen Res. 2015;10(3):377-9.

8. Kamin F, Rommer PS, Abu-Mugheisib M, Koehler W, Hoffmann F, Winkelmann A, Benecke R, Zettl UK. Effects of intrathecal triamincinoloneacetonide treatment in MS patients with therapy-resistant spasticity. Spinal Cord. 2015;53(2):109-13.

9. Rommer PS, Kamin F, Abu-Mugheisib M, Koehler W, Hoffmann F, Winkelmann A, Benecke R, Zettl UK. Long-term effects of repeated cycles of intrathecal triamcinolone acetonide on spasticity in MS patients. CNS Neurosci Ther. 2016;22(1):74-9.

10. Pitarokoili K, Kohle F, Motte J, Fatoba O, Pedreiturria X, Gold R, Yoon MS Anti-inflammatory and immunomodulatory potential of human immunoglobulin applied intrathecally in Lewis rat experimental autoimmune neuritis. J Neuroimmunol. 2017:309:58-67.

11. Hughes RA, Pritchard J, Hadden RD. Pharmacological treatment other than corticosteroids, intravenous immunoglobulin and plasma exchange for Guillain-Barré syndrome. Cochrane Database Syst Rev. 2013;28;(2):CD008630.

12. Kerasnoudis A, Pitarokoili K, Behrendt V, Gold R, Yoon MS. Increased cerebrospinal fluid protein and motor conduction studies as prognostic markers of outcome and nerve ultrasound changes in Guillain-Barré syndrome. J Neurol Sci. 2014;340(1-2):37-43.

13. Makara GB, Haller J. Non-genomic effects of glucocorticoids in the neural system. Evidence, mechanisms and implications. Prog Neurobiol. 2001; 65(4):367-90.

14. Tasker JG, Di S, Malcher-Lopes R. Minireview: rapid glucocorticoid signaling via membrane-associated receptors. Endocrinology. 2006;147(12):5549-56 Epub 2006 Aug 31. Review.

15. Goericke SL, Engelhorn T, Forsting M, Speck U, Maderwald S, Ladd ME, Doerfler A. Intrathecal corticoids in permanent focal cerebral ischemia in rats. Part I: a new therapeutic approach in the acute phase. J Cereb Blood Flow Metab. 2010;30(4):801-7.

16. Enders U, Lobb R, Pepinsky RB, Hartung HP, Toyka KV, Gold R. The role of the very late antigen- 4 and its counterligand vascular cell adhesion molecule- 1 in the pathogenesis of experimental autoimmune neuritis of the Lewis rat. Brain. 1998;121(Pt 7):1257-66.

17. Fairbanks CA. Spinal delivery of analgesics in experimental models of pain and analgesia. Adv Drug Deliv Rev. 2003;55(8):1007-41 Review.

18. Tuck RR, Antony JH, McLeod JG. F-wave in experimental allergic neuritis. J Neurol Sci. 1982;56(2-3):173-84
19. Taylor JM, Pollard JD. Neurophysiological changes in demyelinating and axonal forms of acute experimental autoimmune neuritis in the Lewis rat. Muscle Nerve. 2003;28(3):344-52.

20. Andersen ND, Srinivas S, Piñero G, Monje PV. A rapid and versatile method for the isolation, purification and cryogenic storage of Schwann cells from adult rodent nerves. Sci Rep. 2016:6:31781.

21. Gold R, Toyka KV, Hartung HP. Synergistic effect of IFN-gamma and TNFalpha on expression of immune molecules and antigen presentation by Schwann cells. Cell Immunol. 1995:165(1):65-70.

22. Pfaffl MW. A new mathematical model for relative quantification in real-time RT-PCR. Nucleic Acids Res. 2001:29(9):e45.

23. Demicheva E, Cui YF, Bardwell P, Barghorn S, Kron M, Meyer AH, Schmidt M Gerlach B, Leddy M, Barlow E, O'Connor E, Choi CH, Huang L, Veldman GM, Rus H, Shabanzadeh AP, Tassew NG, Monnier PP, Müller T, Calabresi PA, Schoemaker $\mathrm{H}$, Mueller BK. Targeting repulsive guidance molecule A to promote regeneration and neuroprotection in multiple sclerosis. Cell Rep. 2015;10(11):1887-98.

24. Hayashi R, Xiao W, Kawamoto M, Yuge O, Bennett GJ. Systemic glucocorticoid therapy reduces pain and the number of endoneurial tumor necrosis factoralpha (TNFalpha)-positive mast cells in rats with a painful peripheral neuropathy. J Pharmacol Sci. 2008;106(4):559-65 Epub 2008 Apr 3.

25. Shimizu F, Sawai S, Sano Y, Beppu M, Misawa S, Nishihara H, Koga M, Kuwabara S, Kanda T. Severity and patterns of blood-nerve barrier breakdown in patients with chronic inflammatory demyelinating polyradiculoneuropathy: correlations with clinical subtypes. PLoS One. 2014; 9(8):e104205.

26. Brattsand R, Linden M. Cytokine modulation by glucocorticoids: mechanisms and actions in cellular studies. Aliment Pharmacol Ther. 1996; 10(Suppl 2):81-90 discussion 91-2. Review.

27. Neuberger TJ, Kalimi O, Regelson W, Kalimi M, De Vries GH. Glucocorticoids enhance the potency of Schwann cell mitogens. J Neurosci Res. 1994;38(3): 300-13.

28. Groyer G, Eychenne B, Girard C, Rajkowski K, Schumacher M, Cadepond F. Expression and functional state of the corticosteroid receptors and 11 betahydroxysteroid dehydrogenase type 2 in Schwann cells. Endocrinology. 2006;147(9):4339-50

29. Chan JR, Phillips $\sqcup$ 2nd, Glaser M. Glucocorticoids and progestins signal the initiation and enhance the rate of myelin formation. Proc Natl Acad Sci U S A. 1998;95(18):10459-64.

30. Désarnaud F, Bidichandani S, Patel PI, Baulieu EE, Schumacher M. Glucocorticosteroids stimulate the activity of the promoters of peripheral myelin protein-22 and protein zero genes in Schwann cells. Brain Res. 2000; 865(1):12-6.

31. Morisaki S, Nishi M, Fujiwara H, Oda R, Kawata M, Kubo T. Endogenous glucocorticoids improve myelination via Schwann cells after peripheral nerve injury: an in vivo study using a crush injury model. Glia. 2010;58(8):954-63.

32. Zhang H, Shao Z, Zhu Y, Shi L, Li Z, Hou R, Zhang C, Yao D. Toll-like receptor 4 (TLR4) expression affects Schwann cell behavior in vitro. Sci Rep. 2018;8(1):11179.

33. Skundric DS, Lisak RP, Rouhi M, Kieseier BC, Jung S, Hartung HP. Schwann cellspecific regulation of IL-1 and IL-1Ra during EAN: possible relevance for immune regulation at paranodal regions. J Neuroimmunol. 2001;116(1):74-82.

34. Müller T, Herrling T, Lütge $S$, Lohse L, Öhm G, Jung K. One-time intrathecal triamcinolone acetonide application alters the redox potential in cerebrospinal fluid of progressive multiple sclerosis patients: a pilot study. Ther Adv Neurol Disord. 2016;9(4):264-8.

35. Rommer PS, Kamin F, Petzold A, Tumani H, Abu-Mugheisib M, Koehler W, Hoffmann F, Winkelmann A, Benecke R, Zettl UK. Effects of repeated intrathecal triamcinolone-acetonide application on cerebrospinal fluid biomarkers of axonal damage and glial activity in multiple sclerosis patients. Mol Diagn Ther. 2014;18(6):631-7.

36. Olmos-Jiménez R, Espuny-Miró A, Díaz-Carrasco MS, Fernández-Varón E, Valderrey-Pulido M, Cárceles-Rodríguez C. Stability of four standardized preparations of methotrexate, cytarabine, and hydrocortisone for intrathecal use. J Oncol Pharm Pract. 2016;22(5):659-65. 